\title{
Using sequential Plan-Do-Study-Act cycles to facilitate implementation of a morbidity and mortality review process
}

\author{
Authors: Amy Davies* and Megan Offer
}

\section{Introduction}

Approximately $50 \%$ of deaths occur in hospital and it is estimated that $3-5 \%$ of these deaths are preventable. ${ }^{1}$ Morbidity and mortality (M\&M) meetings allow these deaths, in addition to expected deaths and cases leading to morbidity to be reviewed.

Implementation of an M\&M process provides an ideal opportunity to use a Plan-Do-Study-Act (PDSA) framework. A PDSA cycle enables small-scale change to occur before widespread implementation of a process. Using sequential PSDA cycles it is possible for stakeholders to feedback throughout the process and adjustments to be made accordingly.

\section{Methods}

An initial proposal outlined details of when and where the meetings would be held (first Monday of each month), selection of patients to be discussed and allocation of patients to presenters. Patients were to be presented by a junior doctor following discussion with a named consultant. For each patient a MS PowerPoint presentation and trust protocol M\&M MS Word pro forma would be completed.

The first, second and third M\&M meetings would act as PDSA cycles in order to develop a reproducible format to the M\&M that all stakeholders had contributed to.

\section{Conclusion}

Implementing an M\&M process has had multiple challenges and the flexibility of the PDSA methodology has enabled the process to be dynamic. Asking those attending for feedback has meant changes are discussed as a department and engagement occurs across all levels instead of being implemented from top-down. Although improvements have been made and the M\&M process is now established further PDSA cycles are required to increase the number of patients discussed.

\section{Reference}

1 Hogan H, Zipfel R, Neuberger ] et al. Avoidability of hospital deaths and association with hospital-wide mortality ratios: retrospective case record review and regression analysis. BMJ 2015;351:h3239.

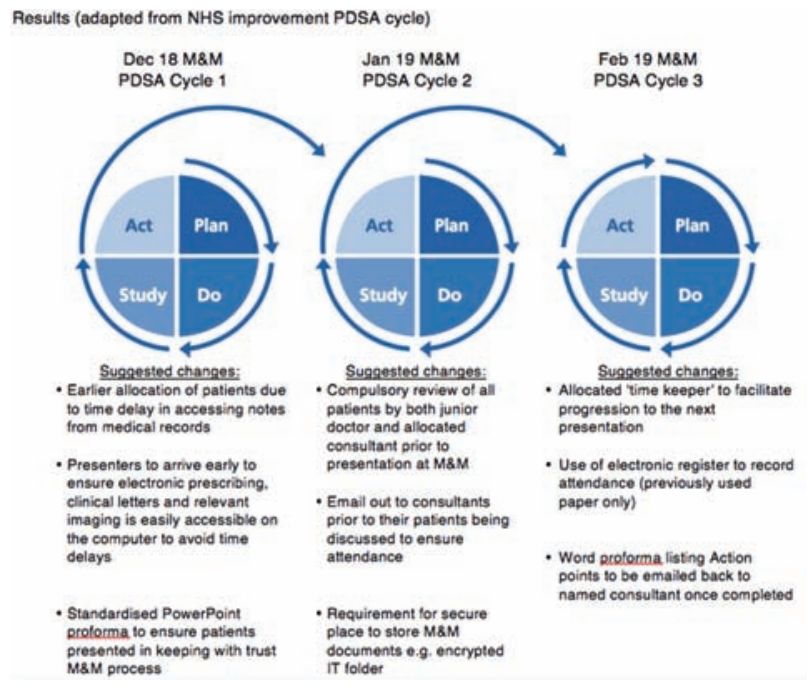

Authors: University Hospitals Birmingham NHS ${ }^{*} \mathrm{RCP}$ chief registrar 